\title{
ANTERIOR SPREAD OF LOCAL ANESTHETIC AFTER THORACO-LUMBAR INTERFASCIAL PLANE BLOCK: A CASE REPORT
}

Giang, Ta, MD

Hanoi Medical University, Hanoi, Vietnam

\section{Introduction}

Thoraco-lumbar inter fascial plane (TLIP) block was first described by Hand et al in 2015 which local anesthetic was injected in the fascial plane between multifidus and longissimus muscle [1]. The TLIP block targets dorsal rami of the thoracolumbar nerves. Clinical studies suggested that TLIP block could be used for analgesia after lumbar spine surgery $[2,3]$. However, there is no data on anterior extension of local anesthetic after TLIP block.

\section{Case study}

Female, 55-year-old patient with herniated disc in the lumbar spine at L4-L5 level was scheduled for elective transforaminal lumbar interbody fusion surgery. The patient had radicular pain on both sides with VAS of 5-6, straight leg raise (SLR) test of $30^{\circ}$ on the left and $45^{\circ}$ on the right side.

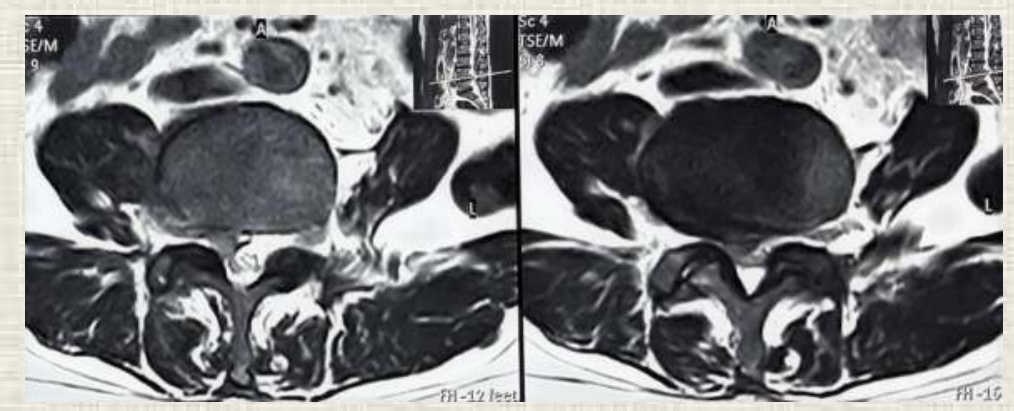

TLIP block was done before general anesthesia. A total of $30 \mathrm{ml}$ Ropivacaine $0.375 \%$ was injected under ultrasound guidance between multifidus and longissimus muscle, bilaterally at level of $L 4$. After block, VAS decreased to 2; SLR test was $>70^{\circ}$ on both sides. The patient reported a reduction of sensation to cold at dermatomes from $L 2$ to $S 1$ at her back and legs.

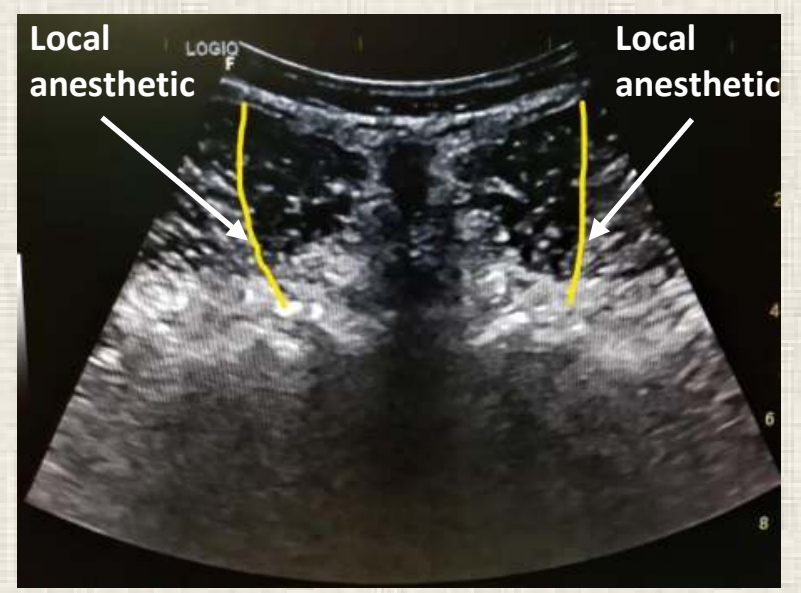

The surgery was done under general anesthesia and lasted for 3 hours. A total of $20 \mathrm{mcg}$ Fentanyl was used intraoperatively.

One gram of Paracetamol and $30 \mathrm{mg}$ of Ketorolac was given postoperatively followed by 2 tablets of Ultracet a day.
The TLIP block lasted for 12 hours. At day 1 after surgery, VAS was 0 at rest and 4 during mobilization. No opioid required postoperatively.

\section{Discussion}

Clinical studies of TLIP block has been published. They showed that TLIP block could provide efficient pain relief after lumbar spine surgery $[2,3]$. However, in all of the studies TLIP block was done after general anesthesia induction and the spread of local anesthetic was not described.

In a cadaveric study of the spread of TLIP block, blue dye was showed to spread to transverse process, and dyed the posterior rami of the lumbar nerves 5 minutes after injection [4]. The anterior spread was not explored.

In this case, the patient reported immediate pain relief, and described a sensory reduction of dermatome from L2 to S1 at her back and legs when we tested with ice 5 minutes after injection. It suggested that the local anesthetic spread anteriorly to block the ventral rami of spinal nerve. We should take it in to consideration if the neuro monitoring is used.

\section{Conclusions}

TLIP block with local anesthetic injection at the fascial plane between multifidus and longissimus muscle can block the lumbar nerve roots and provide radicular pain relief. Anatomical and clinical studies are required to validate the observation.

\section{References}

1. Hand WR, Taylor JM, Harvey NR, Epperson TI, Gunselman RJ, Bolin ED, et al. Thoracolumbar interfascial plane (TLIP) block: a pilot study in volunteers. Can J Anaesth 2015;62:1196-220.

2. Ueshima $H$, Inagaki $M$, Toyone $T$, Otake $H$. Efficacy of the thoracolumbar interfascial plane block for lumbar laminoplasty: a retrospective study. Asian Spine J 2017; 11(5):722-725.

3. Ueshima H, Hara $E$, Otake $H$. Thoracolumbar interfascial plane block provides effective perioperative pain relief for patients undergoing lumbar spinal surgery; a prospective, randomized and double blinded trial. J Clin Anesth 2019; 58: 12-17

4. Ueshima H, Oku K, Otake H. Ultrasound-guided thoracolumbar interfascial plane block: a cadaveric study of the spread of injectate. J Clin Anesth 2016;34:259-60.

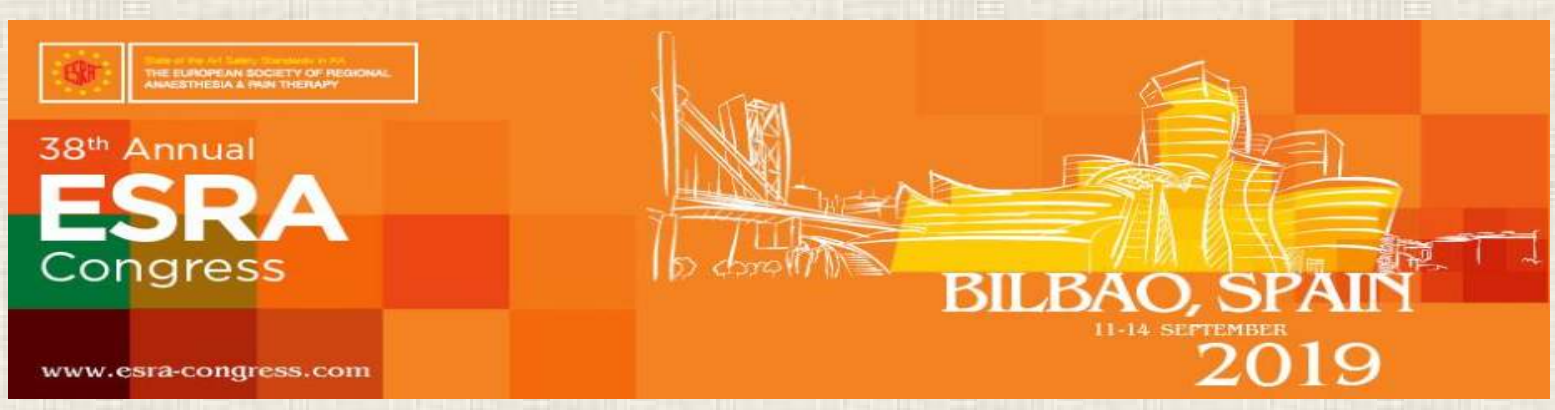

